\title{
The Potential Role of Community Actors in The Response Against COVID-19: Lessons From Three African Countries, Tanzania, Uganda and Zambia
}

Joseph Mumba Zulu

Department of Health Policy and Management, School of Public Health, University of Zambia

Chama Mulubwa

Department of Health Promotion and Education, School of Public Health, University of Zambia

Nathanael Sirili

Department of Developmental Studies, School of Public Health and Social Sciences, Muhimbili University of Health and Allied Sciences, P.O BOX 65454, Dar es Salaam, Tanzania

Adam Silumbwe ( $\square$ adamsilumbwe@gmail.com )

University of Zambia https://orcid.org/0000-0002-1905-5293

\section{Malizgani Paul Chavula}

Department of Health Promotion and Education, School of Public Health, University o Zambia

\section{Margarate Munakampe}

Department of Health Policy and Management, School of Public Health, University of Zambia

\section{Wanga Zulu}

Department of Epidemiology and Biostatistics, School of Public Health, University of Zambia

\section{Charles Michelo}

Department of Epidemiology and Biostatistics, School of Public Health, University of Zambia

Moses Tetui

Department of Health Policy Planning and Management, School of Public Health, University of Makerere

\section{Research note}

Keywords: COVID-19, Community actors, vital, health systems

Posted Date: March 22nd, 2021

DOI: https://doi.org/10.21203/rs.3.rs-322266/v1

License: (c) (1) This work is licensed under a Creative Commons Attribution 4.0 International License.

Read Full License 


\section{Abstract}

\section{Objective}

With the spread of COVID-19 to most low-and middle- income countries, global concerns arise on how to respond to the pandemic. We seek to highlight the early response to COVID-19 of Tanzania, Uganda and Zambia and draw lessons on how community actors could be engaged in the global efforts to prevent its spread and resurgence. This is envisioned to guide COVID-19 prevention efforts as well as implementation of interventions, especially in areas with relaxed, no or partial lockdown measures.

\section{Results}

Community actors can be useful in the promotion of behavioural change including consistent use of face masks, handwashing, social distancing, as well as act as whistle-blowers who identify new residents, report suspected COVID-19 cases and those breaking self-quarantine directives. Furthermore, community actors can encourage adherence to government directives on COVID-19 prevention through integrating COVID-19 information into their routine services. Countries across the globe have the opportunity to tap into the potential role of community actors, especially as we move towards more inclusive health systems. Increased involvement of community health systems is vital in sustaining the gains that have been made in areas where COVID-19 cases have reduced.

\section{Introduction}

COVID-19 is a rapidly evolving global health pandemic[1]. Experiences from other highly affected countries such as the United States of America (USA), Spain and Italy have shown that the virus can be devastating. Albeit, advanced healthcare systems, the pandemic took many of these countries by surprise, thereby making its containment even more challenging [2]. With the spread of COVID-19 to most low-and middle- income countries (LMICs), global concerns arise on how to respond to the pandemic[3], particularly in Africa where health systems are fragile [4]. In this perspective, we aim to highlight the response of Tanzania, Uganda and Zambia and draw lessons on how community actors could be engaged in the global fight against COVID-19. Community actors may include local opinion leaders such as politicians, artists, heads of religious or other social groupings, celebrities, community health workers and village health teams (VHTs).

Over the last decades, LMICs have recorded various health gains that have been linked to major contributions by community level actors [5]. Many LMICs have relied on community-based actors to effectively deliver maternal, newborn, child health, malaria and HIV/AIDS interventions among marginalized and hard to reach populations [6]. The use of community actors is not unique to LMICs, high income countries like Sweden are using community actors to activate virtual health rooms for rural communities. In the USA and Australia, the use of community actors to deal with high risk group behavior change has equally been reported [7]. The collective use of community resources to create better health for all has been termed as reactivating the community health systems by scholars [8]. A community 
health system is defined as a set of local actors, relationships, and processes engaged in producing, advocating for, and supporting health in communities, but existing in relationship to established health structures [9].

The focus on community health systems in the era of COVID-19 is of paramount importance as reflected in the Policy Brief issued by the World Health Organisation (WHO) on $1^{\text {st }}$ April 2020, which outlined 16 recommendations for strengthening health system response to COVID-19 [10]. In this Brief, it was stated that the early experience in countries with large-scale community transmission showed that COVID-19 required unprecedented mobilization of health systems. The first recommendation that health systems ought to consider is expanding their capacity for communication of COVID-19 related information and proactively manage information flow. The use of community actors to supplement such communication therefore comes in handy, and is critical to tackling the widespread miss-information on COVID-19 that is quickly creating another battlefield, referred to as the COVID-19 infodemic by the WHO chief [11].

\section{Main Text}

\section{The COVID -19 response in Tanzania, Uganda and Zambia}

The three African countries generally instituted methods that promote social distancing, although in rather varying ways. While Uganda initially instituted more strict lockdown measures [12,13], Tanzania followed a more "Swedish like" approach. Institutions of learning were closed earlier in the response, while religious gatherings were allowed to continue. Zambia on the other hand instituted measures that lie somewhere in between the two approaches. For instance, gatherings of more than 50 persons were initially banned. The public was largely receptive to lockdown measures in the three countries but left a lot to imagination in places like informal settlements, where measures such as social distancing and frequent handwashing are practically close to impossible. In Kampala for example, $60 \%$ of the population lives in informal settlements where sanitation facilities are shared and living conditions are extremely crowded, making it difficult for residents to social distance or stay locked up in their tiny rooms for extended periods of time [14]. Similarly, in Tanzania and Zambia where lockdowns were not instituted, infection control measures such as regular handwashing and consistent use of face-masks have been more difficult to adopt particularly in lower social economic settings. Therefore, the question of how to reach disadvantaged communities with prevention messages in order to build their capacity to prevent COVID-19 is eminent. Additionally, many rural communities in the three countries are remotely detached from information, and may lack the basic prevention tools such as access to water and soap[15]. The role of community actors therefore becomes even more pertinent given that such settings often rely on them for community sensitizations and mobilization [9]. Indeed, in recognition of the role of community actors, Zambia had started involving a few community volunteers in Lusaka district to sensitise communities on COVID-19 prevention measures as early as mid-April 2020 , hardly a month after the confirmation of the first COVID-19 case. 
Diaconu et al., 2020 have argued that limited community engagement in public health interventions can inadvertently translate into lack of information, potentially leading to trust issues between the government and communities [16]. Without trust, people resort to seeking information and data from unconfirmed and possibly false sources as is now the case with the infodemic. Consequently, leading to myths, misconceptions and low appreciation of government efforts, thus affecting the community's capacity to adhere to COVID-19 preventive measures. In Zambia for example, the Ministry of Health reported that COVID-19 suspects did not avail themselves to the health authorities during contact tracing owing to this mistrust, disinformation and misconceptions. With lockdowns increasingly becoming unsustainable across the globe, finding ways to safely live with the virus becomes critical [17]. We propose that given the prevailing record of community actors in community sensitization and mobilization, tapping into this resource will make a worthwhile contribution to the fight against COVID-19.

\section{Potential roles for community actors}

Evidence suggests that the use of community-based actors to supplement ongoing government efforts to prevent disease spread could potentially help prepare communities to respond better to curb the spread of COVID-19 [18]. Based on lessons from many public health programmes from Tanzania, Uganda and Zambia that have worked with community actors, we believe that community-based actors such as religious leaders, community leaders, traditional healers, teachers, and community health workers (CHWs) can be a critical resource in the fight against COVID-19[19-22]. If well trained and armed with protective clothing and equipment, they could play a significant role in mobilizing communities to adhere to preventive measures by playing the roles discussed in the subsequent paragraphs.

As role models, they promote behavioural change

Several social distancing and infection control measures are being encouraged and made mandatory in some countries; however, community reception and implementation is problematic. We suggest that since community-based health workers, community leaders and other actors such as celebrities are respected and trusted in the communities, if they adopted preventive measures such as wearing of masks, keeping safe distances at shopping malls etc., community adoption of such behaviours could be increased. Similarly, they can play a critical role in delivering culturally sensitive information to counter practices, social norms and misinformation that may propagate the spread of COVID-19 [23]. Lots of misinformation is being spread globally and while governments are doing their best to counter this, community actors could very much enhance these efforts [24]. Celebrities across the globe are already passing on positive messages as is the case of musicians and other artists, whose COVID-19 prevention messages are now on international media making a positive global impact[25].

Acting as whistle blowers toidentify new residents and those breaking self-quarantine directives

Community actors being permanent residents in communities, can easily identify visitors and support enforcement of quarantine directives through relevant bodies [26]. The study by Marais et al., 2016 on the Ebola virus disease (EVD) showed that CHWs using indigenous knowledge in administering safety 
protocols during disease surveillance helped in fostering acceptability of a family member with symptoms to be taken away for admission to a treatment center[27]. The CHWs fostered agreement to voluntary quarantine in special facilities, and greatly diminished stigmatization of survivors of the EVD [28]. Furthermore, working with the community, the $\mathrm{CHWs}$ registered substantial victories through informing people about quarantine measures and in identifying suspected EVD cases. In Zambia, reports of vigilant community leaders have been seen in the fight against home deliveries or deliveries conducted by traditional birth attendants [29].

Working alongside the local leaders to encourage adherence to government directives on COVID-19 prevention

Community-based actors tend to enjoy support from the community leaders, which creates legitimacy for their services[26]. Based on this pre-existing support and legitimacy systems, we note that if properly engaged, community actors can work alongside community leaders to ensure positive reception of government directives such as handwashing and social distancing. Such community collaborations can result in reduced rumors, fear and mistrust[26], which is detrimental to government preventive and curative efforts. Further, such collaboration is key not only in preventing the spread but also re-occurrence of COVID-19 in those countries where the cases have reduced. In fact, as we move towards the discovery and development of vaccines, community acceptance of vaccines will be critical for them to be effective[30]. Therefore, community actors can already start advocating for vaccines to counter the widespread scepticism against vaccines [31]. Historical experience indicates the key role that community actors have played in vaccine acceptance across the globe[30, 32].

Acting as advocates for the continuity of essential health services

In many LMICs, there have been reports of decreasing usage of routine health services such as child immunization and maternal health services due to COVID-19 restrictions/ measures [33]. Community actors such as CHWs and VHTs play a major role in community mobilization and sensitization for a vast majority of health services both preventive and curative in low-income-countries [34]. Harnessing this potential to ensure that they continue carrying out their roles in a safe manner will be a game changer in guaranteeing continuation of health service delivery and usage. This will contribute to the building of resilient health systems. Protective wear, basic training and incentives shall be needed to create and sustain this momentum. For example, reports from Uganda, Tanzania and Zambia indicate that community champions and $\mathrm{CHWs}$ have been essential in creating positive behavioural change regarding uptake of prevention and treatment services in the fight against HIV/AIDS [35-37].

\section{Discussion}

The current call by WHO to strengthen health systems response to COVID-19 and in particular promoting the role of the health system in stopping community spread of COVID-19 is timely. From this perspective, we are particularly interested in how the recommendation by WHO on expanding the capacity for communication of COVID-19 related information can be operationalised across the globe given the 
different contextual realities. We suggest that effective use of community actors will be critical in enhancing the benefits of a multifaceted approach to communicating behavioural change messages. Whether it's through the use of celebrities in high income countries or foot soldiers such as CHWs in LMICs, engaging actors and structures that significantly influence their communities will be critical.

Use of the community actors and structures is also important as it may trigger collective action and responsibility towards addressing COVID-19 [38]. This may among other aspects create a better appreciation of the non-discriminatory nature of the pandemic and therefore calling for a consorted fight to defeat it. The use of community structures and actors as collectively envisioned in the definition of community health systems, creates opportunities for harnessing community resources for the good of society's health and wellbeing. In doing so, it's critical that the community actors are equipped with the right information and tools to stimulate the needed changes.

\section{Conclusion}

The use of community actors to back drop the formal health system is a long-standing global tradition. The re-energizing of such community resources in the fight against COVID-19 will expand the needed resources and contribute towards the building of resilient health systems. Additionally, it will trigger a sense of community and common responsibility, which according to WHO is critical in the fight against COVID-19 unlike polarizing acts that have so far dominated the global COVID-19 response[13].

\section{Limitations}

The lack of primary data is a major limitation to this study. However, the multi-country nature, wide literature review as well as author experiences on the topic strengthens the paper's contribution to knowledge.

\section{Declarations}

The authors alone are responsible for the views expressed in this article and they do not necessarily represent the views, decisions or policies of the institutions with which they are affiliated.

\section{- Ethics approval and consent to participate}

Not applicable

\section{Consent for publication}

Not applicable

\section{- Availability of data and material}

Not applicable 


\section{- Competing interests}

The authors declare that they have no competing interests.

\section{- Funding}

No funding was received for this study

\section{- Authors' contributions}

The study was conceived and designed by JMZ, MT, CM and NS. AS, MPC, MM, WZ, CM conducted the literature review and drafted the manuscript. All authors critically reviewed, revised and edited the draft manuscript. All authors read and approved the final manuscript.

\section{- Acknowledgements}

The authors would like to acknowledge the three institutions, that is University of Makeree, University of Zambia and Muhimbili University for facilitating and ensuring that this collaboration was made possible.

\section{References}

1. Zunyou Wu and Jennifer M McGoogan, Characteristics of and important lessons from the coronavirus disease 2019 (COVID-19) outbreak in China: summary of a report of 72314 cases from the Chinese Center for Disease Control and Prevention. Jama, 2020.

2. Helena Legido-Quigley, et al., Are high-performing health systems resilient against the COVID-19 epidemic? The Lancet, 2020. 395(10227): p. 848-850.

3. Marius Gilbert, et al., Preparedness and vulnerability of African countries against importations of COVID-19: a modelling study. The Lancet, 2020. 395(10227): p. 871-877.

4. Margaret E Kruk, et al., What is a resilient health system? Lessons from Ebola. The Lancet, 2015. 385(9980): p. 1910-1912.

5. Sarah Wood Pallas, et al., Community health workers in low-and middle-income countries: what do we know about scaling up and sustainability? American journal of public health, 2013. 103(7): p. e74-e82.

6. Joseph Mumba Zulu, et al., Integrating national community-based health worker programmes into health systems: a systematic review identifying lessons learned from low-and middle-income countries. BMC public health, 2014. 14(1): p. 987.

7. Henry B Perry, Rose Zulliger, and Michael M Rogers, Community health workers in low-, middle-, and high-income countries: an overview of their history, recent evolution, and current effectiveness. Annual review of public health, 2014. 35: p. 399-421.

8. Asha S George, et al., Hubris, humility and humanity: expanding evidence approaches for improving and sustaining community health programmes. BMJ Global Health, 2018. 3(3). 
9. Helen Schneider and Uta Lehmann, From community health workers to community health systems: time to widen the horizon? Health Systems \& Reform, 2016. 2(2): p. 112-118.

10. WHO., Strengthening the health system response to COVID-19 Recommendations for the WHO European Region Policy brief. 13 May 2020; Available from: http://www.euro.who.int/_data/assets/pdf_file/0003/436350/strengthening-health-systemresponse-COVID-19.pdf?ua=1.

11. John Zarocostas, How to fight an infodemic. The Lancet, 2020. 395(10225): p. 676.

12. Moses Tetui Phillip Wanduru, Peter Waiswa.COVID-19 response in Uganda: notes and reflections.BMJ Global Health Blog.2 May 2020.https://blogs.bmj.com/bmjgh/2020/05/02/covid19-response-in-uganda-notes-and-reflections/

13. Moses Tetui.Coronavirus in East Africa.ThinkGlobalHealth. 29 April 2020.https://www.thinkglobalhealth.org/article/coronavirus-east-africa

14. Innocent Kamara Tumwebaze, et al., Sanitation facilities in Kampala slums, Uganda: users' satisfaction and determinant factors. International journal of environmental health research, 2013. 23(3): p. 191-204.

15. Billie Bonevski, et al., Reaching the hard-to-reach: a systematic review of strategies for improving health and medical research with socially disadvantaged groups. BMC medical research methodology, 2014. 14(1): p. 42.

16. Karin Diaconu, et al., Understanding fragility: implications for global health research and practice. Health policy and planning, 2020. 35(2): p. 235-243.

17. Scott R Baker, et al., Covid-induced economic uncertainty2020. 23 April 2020; Available from: https://retirementincomejournal.com/wp-content/uploads/2020/05/NBER-Covid-InducedUncertainty-5-20.pdf.

18. Henry B Perry, et al., Community health worker programmes after the 2013-2016 Ebola outbreak. Bulletin of the World Health Organization, 2016. 94(7): p. 551.

19. Gertrude Namazzi, et al., Working with community health workers to improve maternal and newborn health outcomes: implementation and scale-up lessons from eastern Uganda. Global health action, 2017. 10(sup4): p. 1345495.

20. Irene A Lema, et al., Community health workers to improve antenatal care and PMTCT uptake in Dar es Salaam, Tanzania: a quantitative performance evaluation. Journal of acquired immune deficiency syndromes (1999), 2014. 67(Suppl 4): p. S195.

21. Adam Silumbwe, Hikabasa Halwindi, and Joseph Mumba Zulu, How community engagement strategies shape participation in mass drug administration programmes for lymphatic filariasis: The case of Luangwa District, Zambia. PLoS neglected tropical diseases, 2019. 13(11): p. e0007861.

22. Helen Mwiinga Chipukuma, et al., Towards a framework for analyzing determinants of performance of community health workers in malaria prevention and control: a systematic review. Human resources for health, 2018. 16(1): p. 22. 
23. E Lee Rosenthal, et al., Community health workers: part of the solution. Health Affairs, 2010. 29(7): p. 1338-1342.

24. Leonardo Bursztyn, et al., Misinformation during a pandemic. University of Chicago, Becker Friedman Institute for Economics Working Paper, 2020(2020-44).

25. Matteo Cinelli, et al., The covid-19 social media infodemic. arXiv preprint arXiv:2003.05004, 2020.

26. Joseph M Zulu, et al., Integrating community health assistant-driven sexual and reproductive health services in the community health system in Nyimba district in Zambia: mapping key actors, points of integration, and conditions shaping the process. Reproductive health, 2019. 16(1): p. 122.

27. Frederick Marais, et al., A community-engaged infection prevention and control approach to Ebola. Health promotion international, 2015. 31(2): p. 440-449.

28. Celso Give, et al., Strengthening referral systems in community health programs: a qualitative study in two rural districts of Maputo Province, Mozambique. BMC health services research, 2019. 19(1): $p$. 263.

29. Chilala Cheelo, Selestine Nzala, and Joseph M Zulu, Banning traditional birth attendants from conducting deliveries: experiences and effects of the ban in a rural district of Kazungula in Zambia. BMC pregnancy and childbirth, 2016. 16(1): p. 323.

30. Adrienne Guignard, et al., Introducing new vaccines in low-and middle-income countries: challenges and approaches. Expert review of vaccines, 2019. 18(2): p. 119-131.

31. Mohamed $F$ Jalloh, et al., Mobilize to vaccinate: lessons learned from social mobilization for immunization in low and middle-income countries. Human vaccines \& immunotherapeutics, 2019: p. $1-7$.

32. Severin Kabakama, et al., Social mobilisation, consent and acceptability: a review of human papillomavirus vaccination procedures in low and middle-income countries. BMC Public Health, 2016. 16(1): p. 834.

33. Lucia Rocca-Ihenacho and Cristina Alonso, Where do women birth during a pandemic? Changing perspectives on Safe Motherhood during the COVID-19 pandemic. Journal of Global Health Science, 2019. 2.

34. Mary-Beth Malcarney, et al., The changing roles of community health workers. Health services research, 2017. 52: p. 360-382.

35. Stephen Asiimwe, et al., Expanding HIV testing and linkage to care in southwestern Uganda with community health extension workers. Journal of the International AIDS Society, 2017. 20: p. 21633.

36. Aurélie Brunie, et al., Integrating family planning and HIV services at the community level: formative assessment with village health teams in Uganda. African Journal of Reproductive Health, 2017. 21(1): p. 73-80.

37. Katharine D Shelley, et al., Can volunteer community health workers manage multiple roles? An interrupted time-series analysis of combined HIV and maternal and child health promotion in Iringa, Tanzania. Health policy and planning, 2018. 33(10): p. 1096-1106. 
38. Helen Schneider, et al., The governance of local health systems in the era of Sustainable Development Goals: reflections on collaborative action to address complex health needs in four country contexts. BMJ global health, 2019. 4(3): p. e001645. 